\title{
Reconnection Nanojets in the Solar Corona
}

Patrick Antolin ${ }^{1,2, *}$, Paolo Pagano ${ }^{2}$, Paola Testa $^{3}$, Antonino Petralia $^{4}$, Fabio Reale $^{4,5}$

${ }^{1}$ Department of Mathematics, Physics and Electrical Engineering, Northumbria University, Newcastle Upon Tyne, NE1 8ST, UK

${ }^{2}$ School of Mathematics and Statistics, University of St. Andrews, St. Andrews, Fife KY16 9SS, $U K$

${ }^{3}$ Harvard-Smithsonian Center for Astrophysics, 60 Garden St., Cambridge, MA 02138, USA

${ }^{4}$ INAF-Osservatorio Astronomico di Palermo, Piazza del Parlamento 1, 90134, Palermo, Italy

${ }^{5}$ Dipartimento di Fisica \& Chimica, Università di Palermo, Piazza del Parlamento 1, 90134, Palermo, Italy

The solar corona is shaped and mysteriously heated to millions of degrees by the Sun's magnetic field. It has long been hypothesised that the heating results from a myriad of tiny magnetic energy outbursts called nanoflares driven by the fundamental process of magnetic reconnection. Misaligned magnetic field lines can break and reconnect, producing nanoflares in avalanche-like processes. However, no direct and unique observations of such nanoflares exist to date, and the lack of a smoking gun has cast doubt on the possibility of solving the coronal heating problem. From coordinated multi-band high-resolution observations here we report on the discovery of very fast and bursty nanojets, the telltale signature of reconnection-based nanoflares resulting in coronal heating. Using state-of-the-art numerical simulations we demonstrate that the nanojet is a consequence of the slingshot effect from the 
magnetically tensed, curved magnetic field lines reconnecting at small angles. Nanojets are therefore the key signature to look for reconnection-based coronal heating in action.

\section{Introduction}

Magnetic reconnection in the solar corona relies on the slow photospheric granulation that quasisteadily stresses the magnetic field to produce twisted and braided structures. Decades ago, Parker [1] pioneered in the reconnection scenario and proposed that coronal loops, the building blocks of the solar corona, would form through myriad of tiny reconnection processes in the small misalignments of the braided field lines, each releasing a small amount of energy that is rapidly redistributed by thermal conduction along the reconnected field $[2,3]$. These tiny outbursts of energy were termed nanoflares - intensity bursts on the order of $10^{24} \mathrm{erg}(\approx 9$ orders of magnitude lower than solar flares). This dissipative process goes hand-in-hand with reconnection outflow jets, corresponding to the bi-directional ejection of plasma and plasmoids from the reconnection point, accelerated by the release of magnetic tension to Alfvénic speeds [4, 5, 6].

Localised, small intensity bursts in the nanoflare range associated to coronal heating have been observed in high-resolution Extreme Ultra-Violet (EUV) [7, 8], Ultra-Violet (UV) [9, 10, 11] and visible observations [e.g. 12]. Non-flare related, high temperatures of 10 million degrees have also been indirectly inferred in X-ray observations of coronal loops and attributed to nanoflares [13]. However, no reports so far have been able to directly link and unequivocally associate a coronal nanoflare-like intensity burst to a magnetic reconnection event leading to coronal heating, as initially proposed by Parker. This challenge has been considered unfeasible since the dissipation 
scales of the current sheets where reconnection occurs are impossible to detect. Moreover, while initially attributed solely to reconnection, nanoflare-like intensity bursts have since been obtained in non-reconnection based numerical models, such as wave heating [14, 15], leading to a lack of a direct observational diagnostic allowing to differentiate coronal heating mechanisms at the smallest discernible scales.

Instead, observational evidence for magnetic reconnection in the solar corona has been provided based on large-scale changes in the magnetic field following reconnection, large plasmoids in long current sheets typical of solar flares or in filament eruptions [16], large-scale plasma outflow at Alfvénic speeds along the reconnected magnetic field [17, 18, 19], and at small-scales in low-lying photospheric [20] and chromospheric structures [21] often leading to field-aligned jets $[22,23]$. The absence of direct observational evidence of small-scale, in-situ dissipation in the solar corona has cast doubt on the possibility to solve the coronal heating problem in the foreseeable future.

A collection of nanoflares (known as a nanoflare storm) due to reconnection can provide substantial coronal heating only if they are triggered once the stressed field loads enough free energy to heat the loop. A popular theory for the switch-on mechanism is the MHD avalanche model: the system reaches a critical state in which the local loss of equilibrium in an elemental loop structure (strand) propagates to the entire structure, producing a nanoflare storm that heats the entire loop [24, 25, 26]. To date, no reports exist of an MHD avalanche of nanoflares leading to the formation of a coronal loop. 


\section{Analysis}

In this work we report the discovery of nanojets, a reconnection-based nanoflare telltale signature that accompany the coronal heating process of a loop to multi-million degree temperatures, building a multi-wavelength band coherent scenario. The spatial and temporal evolution of the heating events and the dynamics of the braided loop structure bear characteristics that can be attributed to an MHD avalanche.

Coordinated observations with the Atmospheric Imaging Assembly (AIA) [27] of the Solar Dynamics Observatory (SDO), the Interface Region Imaging Spectrograph (IRIS) [28] and the Hinode/Solar Optical Telescope (SOT) [29, 30] were carried out on April 3rd, 2014 with target a looplike structure at the limb of the Sun. The loop presented a dip at the apex, hosting a high standing prominence at a height of 20,000 km to 40,000 km (see Fig. 1 and Extended Data Fig.8). From the top of the structure the material flowed as coronal rain along curved paths to the solar surface continuously during several hours, allowing the dynamic and minute tracing of the magnetic field. The off-limb observation presents a dark background to the loop structure and a sideways line-of-sight (LOS) to the loop plane [31]. This preferential configuration has allowed a clear distinction of the dynamics. Several rain strands were observed, and particularly in the $2796 \AA$ and $1400 \AA$ A SJI filters of IRIS, indicating temperatures from 10,000 K to 100,000 K [ref. 28]. The strands presented small apparent misalignments in the plane-of-the-sky (POS) with maximum angles of $25^{\circ} \pm 5^{\circ}$. The EUV absorption from the rain suggests densities of $1.6 \times 10^{10}-1.6 \times 10^{11} \mathrm{~cm}^{-3}$, typical of coronal rain [32] (Supplementary Information). The loop structure was initially only dimly visible 
in the EUV $171 \AA$ A channel of AIA, indicating mild heating to 700, $000 \mathrm{~K}$ temperatures.

The focus of this work is on the last $30 \mathrm{~min}$ of observation. The loop started an accelerated and differential expansion up to $15 \mathrm{~km} \mathrm{~s}^{-1}$, with the apex moving outwards while the visible footpoint remained mostly static. Previous to and, in particular, during the expansion, small and localised intensity bursts are observed in the rain strands. The bursts are accompanied by jet-like structure perpendicular to the rain strands with total speeds of a few hundred $\mathrm{km} \mathrm{s}^{-1}$, followed by rapid outward transverse displacements and rotational motions of the strands up to $60 \mathrm{~km} \mathrm{~s}^{-1}$ (Figs. 2 and 3, see also Extended Data Figs. 1, 2 and Supplementary Figs. 2-9). These jets that we term nanojets are best visible in the transition region IRIS $1400 \AA$ channel, they have EUV signatures in most AIA channels and present almost no signature in the chromospheric IRIS 2796 Å channel (Extended Data Figs. 3, 4 and Supplementary Figs. 10-12). They have widths on the order of $500 \mathrm{~km}$, lengths of 1,000 km-2, $000 \mathrm{~km}$ and are extremely short lived ( $\approx 15$ s or less). Most are only visible for one snapshot in IRIS $1400 \AA$, but can be followed at higher cadence with AIA, indicating POS speeds of $100 \mathrm{~km} \mathrm{~s}^{-1}-200 \mathrm{~km} \mathrm{~s}^{-1}$. The most prominent set of nanojets occurs at the beginning of the expansion. The set is highly clustered near the apex, at the lower part of the loop, with some nanojets separated by $1^{\prime \prime}$ or less (Fig. 2). The episode lasts about $100 \mathrm{~s}$ and brings the largest morphological change within the loop. Subsequently, other nanojets appear throughout the loop, some occurring in clusters and others in apparent isolation (Fig. 3). The largest events involve the ejection of plasmoids along the jets axis with widths on the order of $1^{\prime \prime}$ at speeds in the POS of $50 \mathrm{~km} \mathrm{~s}^{-1}-60 \mathrm{~km} \mathrm{~s}^{-1}$ (Fig. 2). Several nanojets were captured by the spectrograph of IRIS and show a LOS velocity component in the Mg II and Si IV lines with 
a magnitude above $100 \mathrm{~km} \mathrm{~s}^{-1}$ and with a spectral line broadening due to unresolved velocities (i.e. non-thermal) of similar magnitude and up to 15 times increase in the Si IV line integrated intensity with respect to the average (e.g. Fig. 4, Table 1). We note that the determination of multiple components in the line profiles is relatively straightforward thanks to the off-limb viewpoint and to the low optical thickness of the rain emission (see Methods).

The spatial distribution of the nanojets spreads out across (inward to outward) and along the loop, with some occurring just above spicular heights (Extended Data Fig. 5). Also, their occurrence increases with time (Extended Data Fig. 6). In the 13 min time span of their occurrence, we estimate the number of resolved nanojets to be on the order of 150 (Extended Data Fig. 7, see Methods). The nanojets precede the formation of coronal strands (Extended Data Fig. 5), which become visible in most EUV wavelengths (e.g. Extended Data Figs. 4 and 8). These are formed locally first, before encompassing, and thereby forming, the coronal loop. The temporal and spatial evolution of the nanojets, the intensity bursts and overall increase of EUV intensity is thus highly suggestive of an MHD avalanche. A differential emission measure (DEM) analysis of the loop with the AIA channels indicates temperatures of $2-5 \mathrm{MK}$, with possible maxima up to 10 MK (Extended Data Fig. 9). The rain strands become in time more parallel to each other in the POS (Extended Data Fig. 10), and show internal rotational motions at speeds of $50 \mathrm{~km} \mathrm{~s}^{-1}$, counter-streaming flows along the loop and non-thermal line broadening both up to $80 \mathrm{~km} \mathrm{~s}^{-1}$ (Supplementary Fig. 13). This is strongly indicative of a reduction in the braiding and of untwisting, expected in MHD avalanche models [e.g. 33]. The dissipated energy per nanojet is estimated to be up to $10^{25}$ erg (Methods), with the bulk of the distribution likely being out of reach for the 
present instrumentation.

To confirm that nanojets with the observed characteristics are indeed possible through reconnection at small-angle magnetic field misalignments and to further examine their nature we conduct a 3D MHD experiment of 2 slightly misaligned flux tubes reconnecting at one point in the corona (Methods). A highly localised reconnection event is obtained which satisfactorily explains the nanojet as one of the bi-directional reconnection outflows and accompanying field line displacement accelerated by magnetic tension (Figure 5). The strongest dynamics (which make up the nanojets) come from the perpendicular advection of the field lines from the reconnection site, with relatively small longitudinal (field-aligned) plasma velocity. Hence, contrary to most reconnection-based solar jets, usually rooted in the chromosphere [e.g. 23], the small-angle coronal reconnection observed here does not involve a strong plasma flow along the reconnected field lines (Figure 6). We see a single nanojet mostly because in a curved loop the magnetic tension is significantly larger inward than outward (Supplementary material). Besides the overall shape and the dynamics, we also recover in the simulations the local temperature increase and the larger-scale perpendicular displacement of the reconnecting field lines (that would characterise a strand in the presence of rain).

Physical drivers for magnetic reconnection can be of many kinds, such as dynamic instabilities or the magnetic field shuffling from granular convection. The major nanojet episodes occur during the loop expansion, and they are likely driven by the partial loss of equilibrium of the prominence at the top of the loop, as suggested by numerical modelling [34] (see Supplementary 
Information). Two other episodes of clustered nanojets are observed much prior to the loop expansion, one in a different loop that may not be attached to the prominence (Supplementary Figs. 16, 17 and 18). These episodes involve the same combination of features: the nanojets, localised intensity bursts and rapid displacement of strands. However, we only observe local variation without large-scale coronal strands, indicating an overall smaller energy release. We therefore conjecture that the nanojet as a singular entity is largely independent of the nature of the driver. As we have shown, their morphology mostly depends on the (small-angle) magnetic reconnection in a strong guide field topology (such as a coronal loop). However, their numbers and evolution as a group (for instance, as an MHD avalanche) will be strongly dependent on the driver, since it defines the overall available energy.

\section{Discussion}

The heating events, the detailed dynamics and morphology, and particularly the details of the nanojets constitute major challenges for reconnection-based numerical models and provide major constraints on the nature of reconnection in the solar corona. In particular, a major finding from these observations is the discrete and very distinct occurrence of the nanojets that, although numerous, appears as a countable phenomenon. This discrete nature may suggest that reconnection occurs episodically and strongly supports the reconnection-based nanoflare theory in that nanoflares constitute elemental heating events that can be responsible for coronal heating. If reconnection were non-localised and continuously occurring along long current sheets the nanojets and the accompanying intensity bursts would not be highly localised but would involve entire rain strands 
moving perpendicularly at Alfvénic speeds (producing a smeared image effect for the displaced rain strands), which is not observed. A possibility is that the rain plays an important role in the reconnection process. The partial ionisation state of the rain means that ambipolar diffusion is more efficient, thereby facilitating reconnection [35]. However, not all of the observed nanojets appear rooted in cool and dense plasma, as the high resolution Hinode/SOT observations indicate. Also, most nanojets cannot be seen in the chromospheric channels (see Methods). Lastly, as our 3D MHD numerical experiment suggests, highly localised reconnection would happen even in the absence of partially ionised and clumpy plasma. This suggests that the rain is a backdrop against which the reconnection nanojets can be seen, and may also be a catalyst of the process but not a requirement.

Another theoretical challenge is the appearance of plasmoids accompanying the nanojets at large enough scales to be visible with present instrumentation, suggesting that the magnetic islands involved in the secondary tearing mode instability can grow in non-flare related and small-scale current sheets. We therefore expect that the nanojets resolved here constitute the high end of the true distribution of spatial and energy scales involved in this reconnection scenario, so that the bulk of the distribution has energies below $10^{25} \mathrm{erg}$, as predicted from theory [1]. Our results therefore strongly suggest that next-generation instrumentation will readily observe the distribution of scales of these events. The distinct observational signatures revealed here serve as a guide that allows to distinguish heating mechanisms based on magnetic reconnection, thus providing a clear target and path for next-generation high-resolution instrumentation to solve the coronal heating problem. 


\section{Main References}

1. Parker, E. N. Nanoflares and the solar X-ray corona. Astrophys. J. 330, 474-479 (1988).

2. Cargill, P. J. Some implications of the nanoflare concept. Astrophys. J. 422, 381-393 (1994).

3. Rappazzo, A. F., Velli, M., Einaudi, G. \& Dahlburg, R. B. Nonlinear Dynamics of the Parker Scenario for Coronal Heating. Astrophys. J. 677, 1348-1366 (2008).

4. Biskamp, D. Magnetic reconnection via current sheets. Phys. of Fluids 29, 1520-1531 (1986).

5. Zweibel, E. G. \& Yamada, M. Magnetic reconnection in astrophysical and laboratory plasmas. Annu. Rev. Astron. Astrophys. 47, 291-332 (2009).

6. Bhattacharjee, A., Huang, Y.-M., Yang, H. \& Rogers, B. Fast reconnection in high-lundquistnumber plasmas due to the plasmoid instability. Phys. of Plasmas 16, 112102 (2009).

7. Cirtain, J. W. et al. Energy release in the solar corona from spatially resolved magnetic braids. Nature 493, 501-503 (2013).

8. Testa, P. et al. Observing Coronal Nanoflares in Active Region Moss. Astrophys. J. Lett. 770, L1 (2013).

9. Testa, P. et al. Evidence of nonthermal particles in coronal loops heated impulsively by nanoflares. Science 346, 1255724 (2014).

10. Testa, P., Polito, V. \& Pontieu, B. D. IRIS Observations of Short-term Variability in Moss Associated with Transient Hot Coronal Loops. Astrophys. J. 889, 124 (2020). 
11. Tian, H. et al. Observations of Subarcsecond Bright Dots in the Transition Region above Sunspots with the Interface Region Imaging Spectrograph. Astrophys. J. Lett. 790, L29 (2014).

12. Bai, X. Y. et al. Multi-wavelength Observations of a Subarcsecond Penumbral Transient Brightening Event. Astrophys. J. 823, 60 (2016).

13. Ishikawa, S. et al. Detection of nanoflare-heated plasma in the solar corona by the foxsi-2 sounding rocket. Nature Astronomy 1, 771-774 (2017).

14. Moriyasu, S., Kudoh, T., Yokoyama, T. \& Shibata, K. The Nonlinear Alfvén Wave Model for Solar Coronal Heating and Nanoflares. Astrophys. J. Lett. 601, L107-L110 (2004).

15. Antolin, P., Shibata, K., Kudoh, T., Shiota, D. \& Brooks, D. Predicting Observational Signatures of Coronal Heating by Alfvén Waves and Nanoflares. Astrophys. J. 688, 669-682 (2008).

16. Sterling, A. C., Moore, R. L., Falconer, D. A. \& Adams, M. Small-scale filament eruptions as the driver of X-ray jets in solar coronal holes. Nature 523, 437-440 (2015).

17. Takasao, S., Asai, A., Isobe, H. \& Shibata, K. Simultaneous Observation of Reconnection Inflow and Outflow Associated with the 2010 August 18 Solar Flare. Astrophys. J. Lett. 745, L6 (2012).

18. Reale, F., Peres, G., Serio, S., DeLuca, E. E. \& Golub, L. A Brightening Coronal Loop Observed by TRACE. I. Morphology and Evolution. Astrophys. J. 535, 412-422 (2000). 
19. Reale, F. et al. A Brightening Coronal Loop Observed by TRACE. II. Loop Modeling and Constraints on Heating. Astrophys. J. 535, 423-437 (2000).

20. Reid, A. et al. Ellerman Bombs with Jets: Cause and Effect. Astrophys. J. 805, 64 (2015).

21. Rouppe van der Voort, L. et al. Intermittent Reconnection and Plasmoids in UV Bursts in the Low Solar Atmosphere. Astrophys. J. Lett. 851, L6 (2017).

22. Katsukawa, Y. et al. Small-Scale Jetlike Features in Penumbral Chromospheres. Science 318, 1594 (2007).

23. Shibata, K. et al. Chromospheric Anemone Jets as Evidence of Ubiquitous Reconnection. Science 318, 1591 (2007).

24. Lu, E. T., Hamilton, R. J., McTiernan, J. M. \& Bromund, K. R. Solar flares and avalanches in driven dissipative systems. Astrophys. J. 412, 841-852 (1993).

25. Charbonneau, P., McIntosh, S. W., Liu, H.-L. \& Bogdan, T. J. Avalanche models for solar flares (Invited Review). Sol. Phys. 203, 321-353 (2001).

26. Aschwanden, M. J. et al. 25 Years of Self-Organized Criticality: Solar and Astrophysics. Space Sci. Rev. 198, 47-166 (2016).

27. Lemen, J. R. et al. The Atmospheric Imaging Assembly (AIA) on the Solar Dynamics Observatory (SDO). Sol. Phys. 275, 17-40 (2012).

28. De Pontieu, B. et al. The Interface Region Imaging Spectrograph (IRIS). Sol. Phys. 289, 2733-2779 (2014). 
29. Suematsu, Y. et al. The Solar Optical Telescope of Solar-B ( Hinode): The Optical Telescope Assembly. Sol. Phys. 249, 197-220 (2008).

30. Tsuneta, S. et al. The Solar Optical Telescope for the Hinode Mission: An Overview. Sol. Phys. 249, 167-196 (2008).

31. Antolin, P., Pagano, P., De Moortel, I. \& Nakariakov, V. M. In Situ Generation of Transverse Magnetohydrodynamic Waves from Colliding Flows in the Solar Corona. Astrophys. J. Lett. 861, L15 (2018).

32. Antolin, P., Vissers, G., Pereira, T. M. D., Rouppe van der Voort, L. \& Scullion, E. The Multithermal and Multi-stranded Nature of Coronal Rain. Astrophys. J. 806, 81 (2015).

33. Hood, A. W., Cargill, P. J., Browning, P. K. \& Tam, K. V. An MHD Avalanche in a Multithreaded Coronal Loop. Astrophys. J. 817, 5 (2016).

34. Keppens, R. \& Xia, C. The Dynamics of Funnel Prominences. Astrophys. J. 789, 22 (2014).

35. Martínez-Sykora, J. et al. Ion-neutral Interactions and Nonequilibrium Ionization in the Solar Chromosphere. Astrophys. J. 889, 95 (2020).

All correspondence and requests for materials should be addressed to Patrick Antolin.

Acknowledgements P.A. acknowledges STFC support from grants ST/R004285/2 and ST/T000384/1 and support from the International Space Science Institute, Bern, Switzerland to the International Teams on 'Implications for coronal heating and magnetic fields from coronal rain observations and modeling' and 
'Observed Multi-Scale Variability of Coronal Loops as a Probe of Coronal Heating'. This project has received funding from the European Research Council (ERC) under the European Union's Horizon 2020 research and innovation programme (grant agreement No 647214). P.P. would like to thank STFC for IAA funding under grant number SMC1-XAS012. P.T. acknowledges support by 8100002705 and SP02H1701R from Lockheed-Martin to SAO. P.A. would like to thank R. Rutten and I. De Moortel for valuable discussion. Hinode is a Japanese mission developed and launched by ISAS/JAXA, with NAOJ as domestic partner and NASA and STFC (UK) as international partners. It is operated by these agencies in co-operation with ESA and NSC (Norway). IRIS is a NASA small explorer mission developed and operated by LMSAL with mission operations executed at NASA Ames Research Center and major contributions to downlink communications funded by ESA and the Norwegian Space Centre. SDO is part of NASA's Living With a Star Program. All data used in this work is publicly available through the websites of the respective solar missions. This work used the DiRAC Data Centric system at Durham University, operated by the Institute for Computational Cosmology on behalf of the STFC DiRAC HPC Facility. This equipment was funded by a BIS National E-infrastructure capital grant ST/K00042X/1, STFC capital grant ST/K00087X/1, DiRAC Operations grant ST/K003267/1, and Durham University. DiRAC is part of the National E-Infrastructure.

Author contributions statement P.A. was responsible for the planning, coordination, image processing, analysis of the observations and redaction of most of the manuscript. P.P. was responsible for the numerical simulations and redacted the analysis of the numerical results. P.T. was responsible for the differential emission measure analysis and redacted the corresponding section in the manuscript. A.P. and F.R. have provided the numerical code and the initial condition to the numerical simulation. A.P., F.R. and P.T. have also provided feedback on the analysis of the results and the redaction of the manuscript.

Competing interests The authors declare no competing financial interests. 


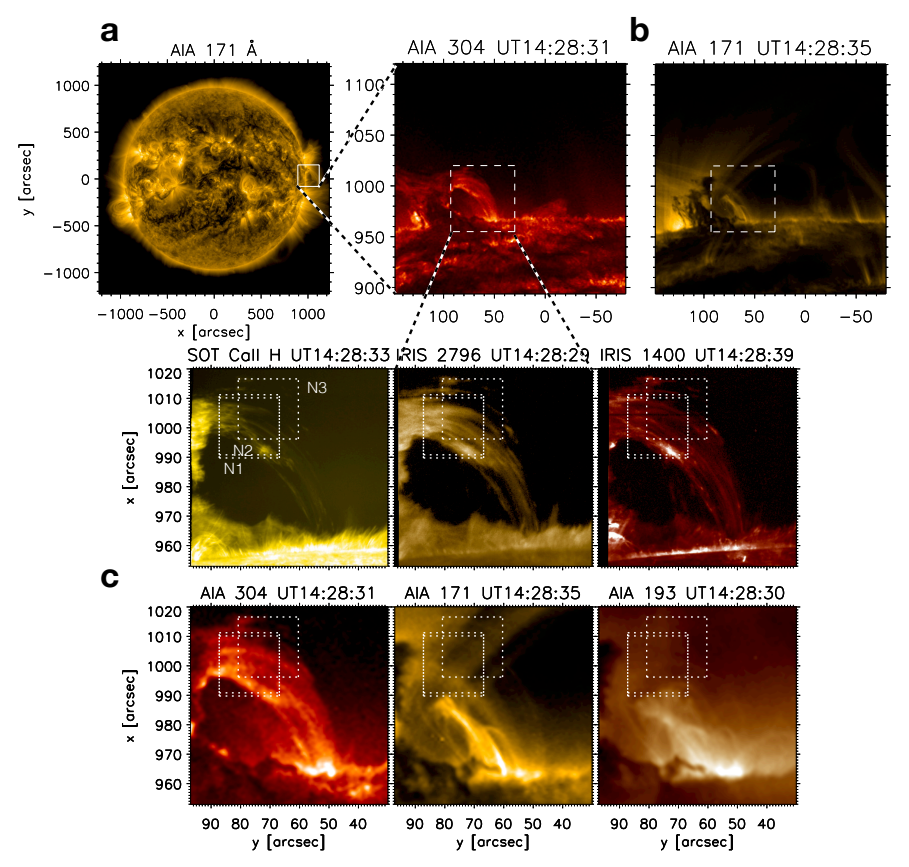

Figure 1: SDO, IRIS and Hinode co-observation of the coronal structure. (Panel a) Full-disk SDO/AIA image in the $171 \AA$ channel of the Sun on April 3rd, 2014. (Panels b) Zoomed and rotated portion of the FOV (white square on the West limb) in the AIA $304 \AA$ and $171 \AA$ A channels. The IRIS/SJI FOV is indicated by the white dashed square. (Panels c, top row) Co-observed FOV between Hinode/SOT (left, in the Ca II H line) and IRIS/SJI (right, in the $2796 \AA$ and $1400 \AA$ A channels, respectively). The visible loop-like coronal structure is the subject of this study. A radial filter has been applied to decrease the intensity of the solar disk and to make the off-limb features more visible. (Panels c, bottom row) The corresponding AIA images for the Hinode and IRIS FOV in the AIA $304 \AA$ A $171 \AA$ and $193 \AA$ channels, respectively from left to right. The main structure of interest is the loop-like structure connecting the prominence (bright structure to the left in the SOT FOV) to the solar surface. The 3 dotted rectangles N1, N2 and N3 within the c panels correspond to the FOVs of Figs. 2, 3 and 4, respectively. 


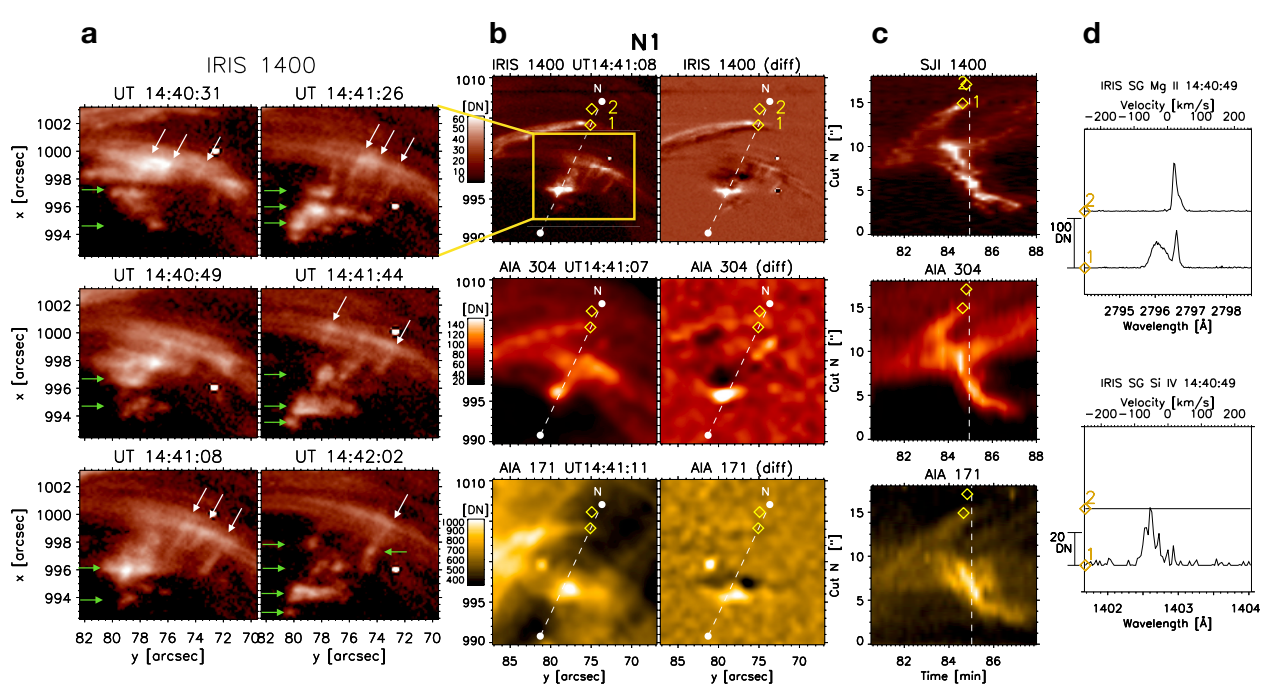

Figure 2: A nanojet cluster with plasmoid ejecta. (Panels a) A snapshot sequence of a nanojet cluster in the IRIS $1400 \AA$ A of a zoomed-in region, as indicated in the figure. Note the tight clustering of the nanojets (white arrows) and the ejection of plasmoid-like structure from the cluster (green arrows). (Panels b) FOV marked 'N1' in Fig. 1 showing a snapshot of the nanojet cluster and its running difference version. From top to bottom rows, the IRIS $1400 \AA$, AIA $304 \AA$ and AIA $171 \AA$ A channels, respectively. The unit of intensity is Data Numbers (DN), proportional to the number of photons $(\approx 1,12$ and $18 \mathrm{ph} / \mathrm{DN}$, respectively, for the AIA channels, IRIS 1400 and 2796). (Panels c) Time-distance diagrams along cut ' $N$ ' (white dashed lines). The time of the snapshot in $\mathbf{b}$ is indicated by the white vertical dashed line. The time is measured from the start of the IRIS observation. (Panels d) The IRIS spectrograph captures part of the process: simultaneous to the ejecta a strand is displaced upward at similar speeds. The 2 yellow diamonds in the IRIS 1400 A images of panels b show slit locations $1 \& 2$ (corresponding to the raster position number) along cut 'N', and their spectra in the Mg II k and Si IV $1402.77 \AA$ lines are shown, respectively, in the top and bottom panels of $\mathbf{d}$ (for the spectrograph we have 4 and $18 \mathrm{ph} / \mathrm{DN}$ for Si IV and Mg II, respectively). We select the pixel with the brightest integrated intensity within a 16 4-pixel distance from cut ' $N$ ' along the slit. An animation of this figure is available online. 


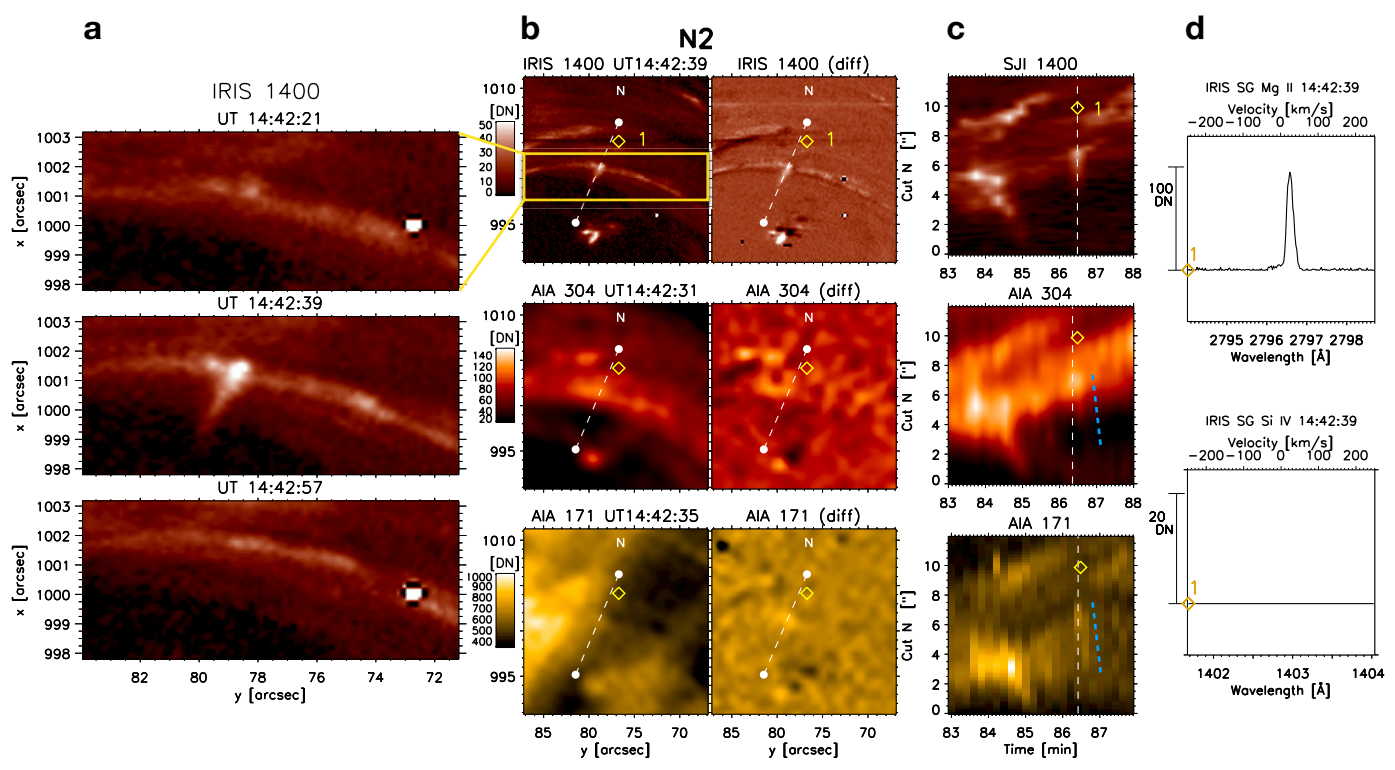

Figure 3: A single nanojet. Keeping the same panel configuration as in Fig. 2, the region marked 'N2' in Fig. 1 is shown here (FOV of panels b). Panels a show a snapshot sequence in the IRIS $1400 \AA$ Af a zoomed-in region in which a single nanojet can be seen. The nanojet is composed of a bright head stemming from a rain strand, a length of $2^{\prime \prime}-3^{\prime \prime}$ and an average width of $500 \mathrm{~km}$. The nanojet is only seen during one snapshot in IRIS $1400 \AA$, but can be followed over a few snapshots in the AIA channels. This produces, respectively, vertical and slanted patterns in the time-distance plots for the IRIS and AIA images (panels c). The slope of the slanted pattern (blue dashed line) indicates a POS speed of $\approx 240 \mathrm{~km} \mathrm{~s}^{-1}$. The IRIS slit is too far from the nanojet and no associated feature can be seen in the spectra (panels d). See Fig. 2 for further explanation of the figure layout. An animation of this figure is available online. 

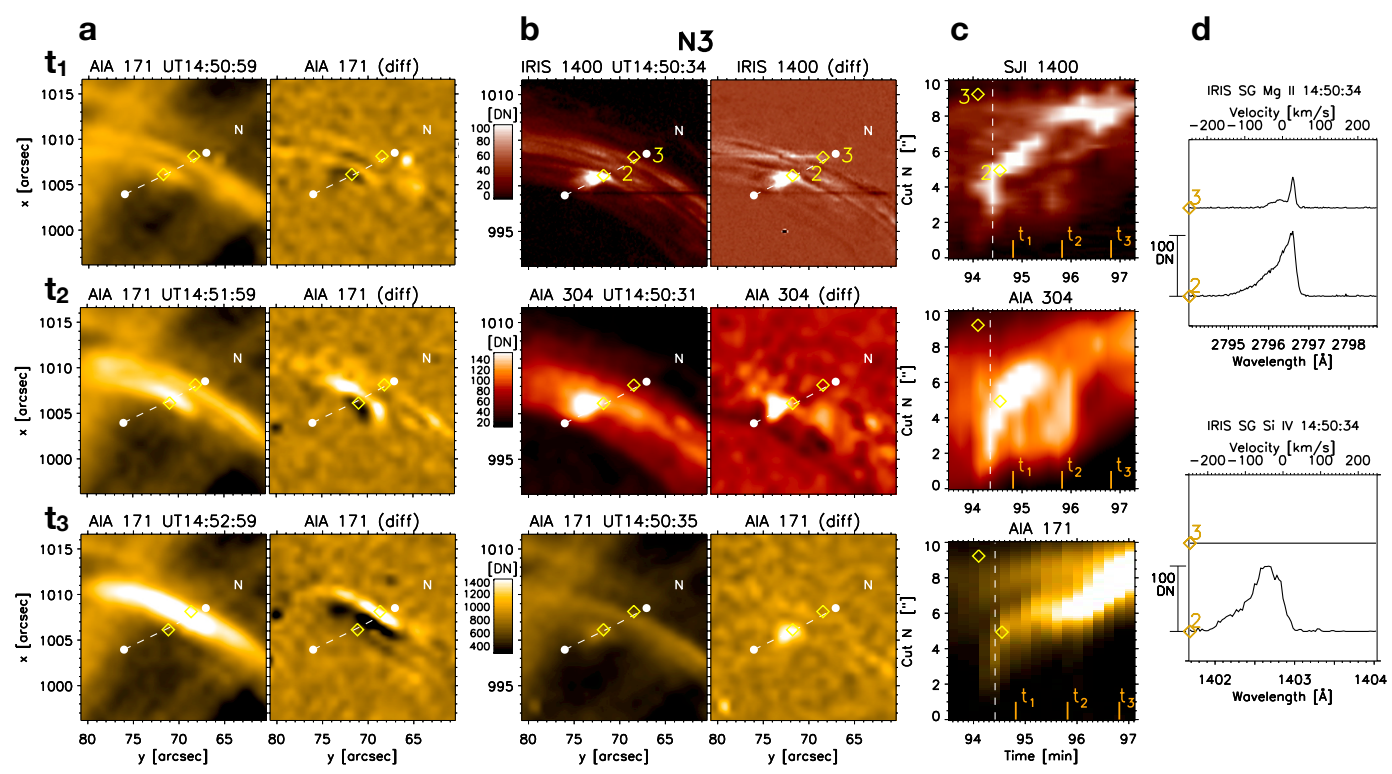

Figure 4: Spectral features of a nanojet and coronal loop formation. The panel configuration of Fig. 2 is kept and we show here a nanojet fully captured by the IRIS slit. Also, panels a show the AIA $171 \AA$ A snapshot pair (of snapshot and its running difference version) for 3 instances in time labeled ' $t_{1}$ ', ' $t_{2}$ ' and ' $t_{3}$ ', each separated by 1 minute, following the snapshot shown in panels b. First, a localised brightening is observed in all channels, whose spectral profiles show blueshifted material with Doppler speeds close to $-200 \mathrm{~km} \mathrm{~s}^{-1}$ (yellow diamond labeled with nr. 2), as seen in panels $\mathbf{d}$. The strand progressively brightens in AIA $171 \AA$, extending along the rain flow. At the same time the strand displaces transversely, upward along cut ' $\mathrm{N}$ '. Note that within the time-distance diagram (panels c) a second nanojet occurs in the strand at the time denoted by ' $\mathrm{t}_{2}$ ', mostly seen in the AIA $171 \AA$ A channel. See Extended Data Fig. 5 for the full spatial extent of the coronal strands. See Fig. 2 for further explanation of the figure layout. An animation of this figure is available online. 

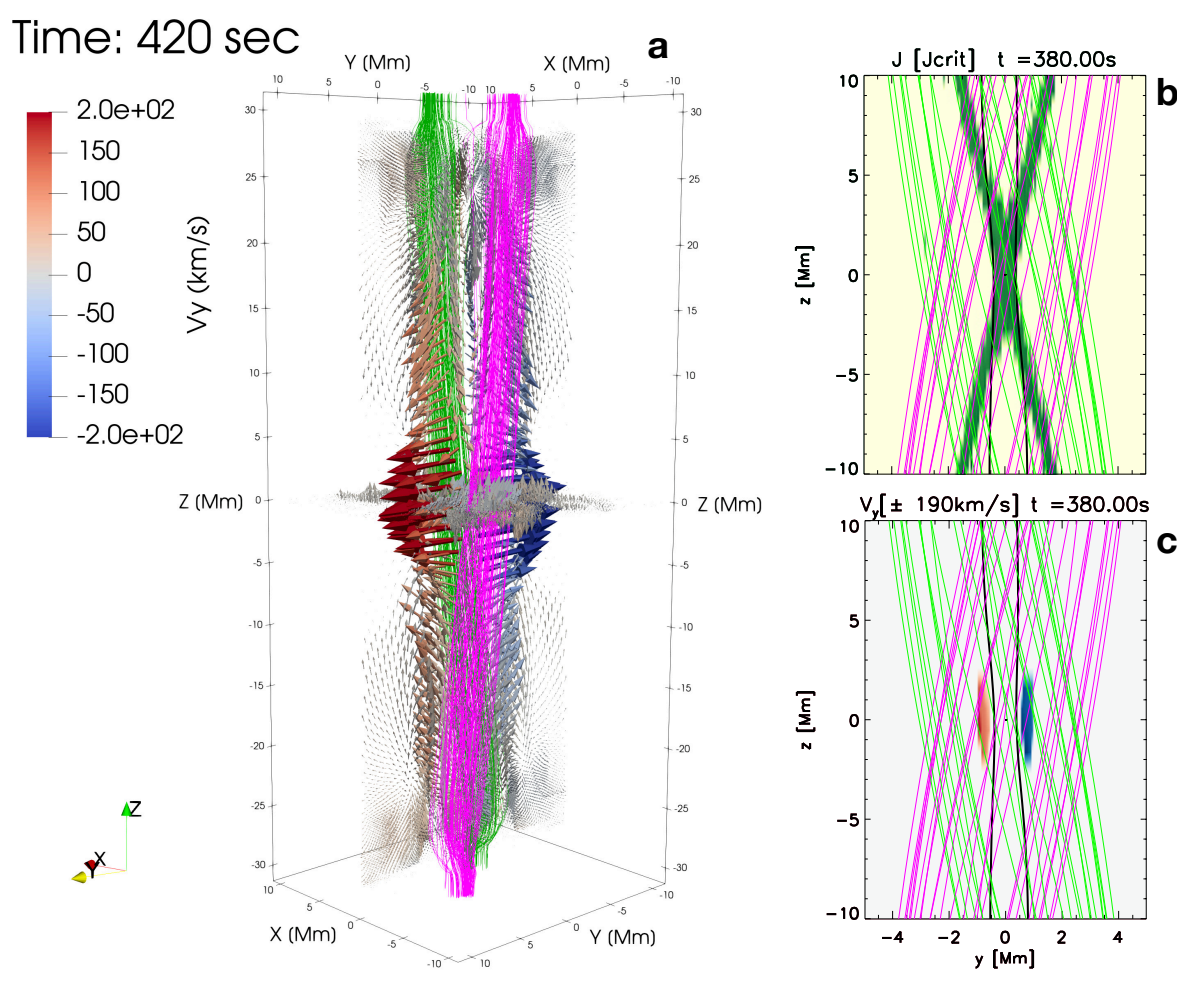

Figure 5: A nanojet in our numerical model. In all panels, magnetic field lines representative of the 2 loops (magenta and green) are displayed, as described in the Methods section. Panel a shows magnetic field lines in the $3 \mathrm{D}$ numerical box at the moment of the nanojet occurrence ( $t=420 \mathrm{~s}$ ). Magnetic reconnection is localised around $z=0$. The magnetic tension from the reconnected magnetic field lines produces a high-velocity (up to $200 \mathrm{~km} \mathrm{~s}^{-1}$ ), bi-directional jet collimated along the $y$-axis. The width of the region with high velocities $\left(>100 \mathrm{~km} \mathrm{~s}^{-1}\right)$ along the $x$ - and $z$-axis is less than $1 \mathrm{Mm}$, and $3 \mathrm{Mm}$ respectively. Note also that the $z$-velocities are only on the order of $20 \mathrm{~km} \mathrm{~s}^{-1}$. In panels $\mathbf{b}$ and $\mathbf{c}$, we zoom-in into the central region at time $t=380 \mathrm{~s}$, just prior to the nanojet, and show the iso-contour of the electric current where $|J|=J_{0}$ (b, X-type green shaded region) and the iso-contours of the $y$-component of the velocity where $v_{y}= \pm 190 \mathrm{~km} \mathrm{~s}^{-1}$ (c, blue/red shaded regions). Note how the region of highest magnetic tension of the reconnected magnetic field lines (in black) match the high-velocity region, leading to the nanojet. An animation of this figure is available online. 


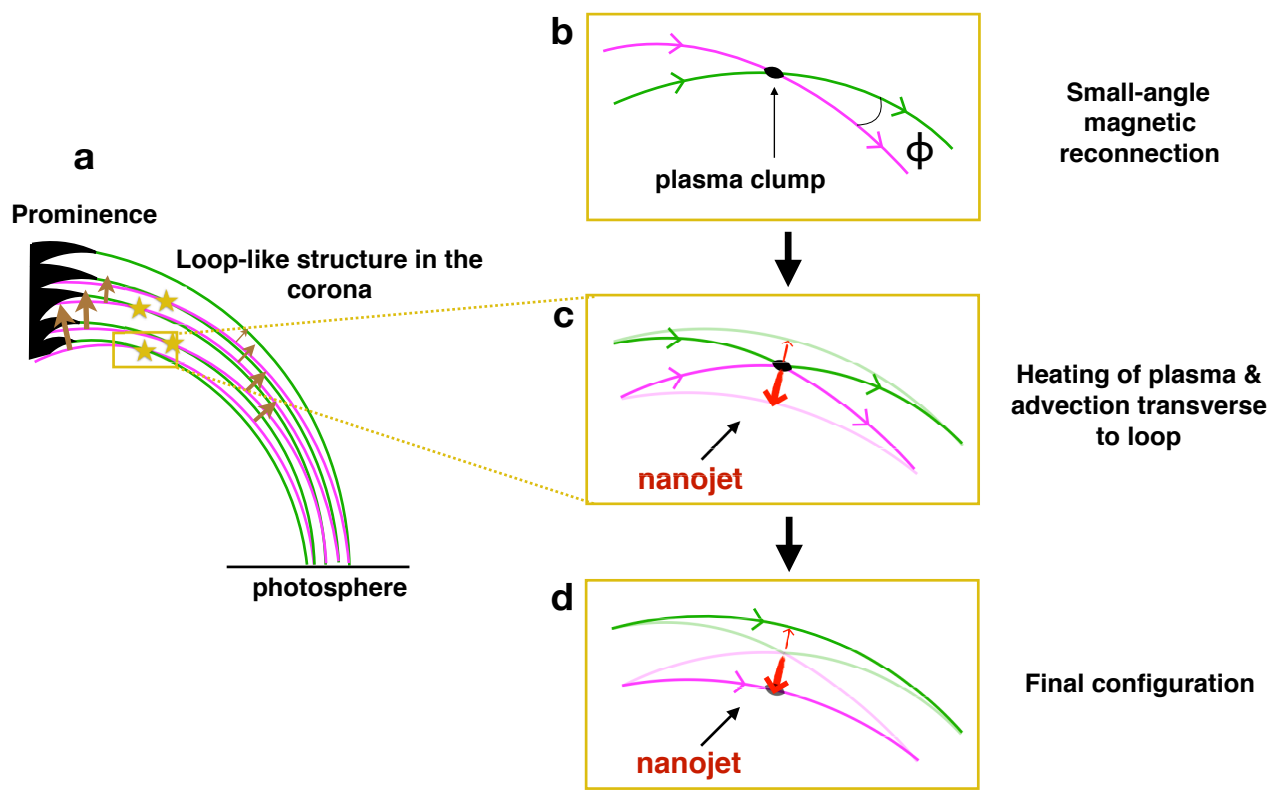

Figure 6: Sketch of a nanojet. (Panel a) Sketch of the loop-like structure as observed in the POS. The brown arrows show the slow upward expansion of the loop apex. The small stars denote the nanoflares, for which the evolution is shown in the zoomed-in $\mathbf{b}, \mathbf{c}$ and $\mathbf{d}$ panels. Small misalignments between green and magenta field lines lead to reconnection at small-angle $\phi$ (panel b). Plasma is heated and advected transversely to the loop at large speeds due to magnetic tension, thereby creating the nanojet (panel c). The inward component is much larger than the outward component due to the curved topology of the field lines, thus leading to the singular nature of the jet. A final configuration is reached with reduced braiding and misalignment between field lines at a higher temperature ( panel d). A coronal strand starts to form. 


\begin{tabular}{|c|c|c|c|}
\hline & & Quiet period (prior to expansion) & nanojets \\
\hline Number & spectra & 7264 & 445 \\
\hline $\begin{array}{c}\text { Total intensity } \\
{[\mathrm{DN}]}\end{array}$ & Mectral components & 7326 & 751 \\
\hline $\begin{array}{c}\sum \mid \text { Dop. vel. } \\
{\left[\mathrm{km} \mathrm{s}^{-1}\right]}\end{array}$ & Standard deviation & 146.2 & 410 \\
\hline $\begin{array}{c}\mid \text { Dop. vel. } \\
{\left[\mathrm{km} \mathrm{s}^{-1}\right]}\end{array}$ & Mean & 28 & 223.4 \\
\hline $\begin{array}{c}\sum \xi \\
{\left[\mathrm{km} \mathrm{s}^{-1}\right]}\end{array}$ & Standard deviation & 15.8 & 103 \\
\hline $\begin{array}{c}\xi \\
{\left[\mathrm{km} \mathrm{s}^{-1}\right]}\end{array}$ & Mean & 10.4 & 36.6 \\
\hline
\end{tabular}

Table 1: Statistics based on Si IV spectra. From top to bottom row we show for the quiet period (prior to expansion) and the nanojets, the number of spectral profiles and spectral components (for each profile), the total (integrated) intensity, the (absolute) Doppler velocity and the non-thermal velocity $\xi$. The sum symbol denotes a sum over all spectral components for each spectral profile. 


\section{Methods}

Spectral line fitting and statistical analysis. The Mg II k and Si IV 1402.77 spectra from the rain present multiple components, and both broad and thin spectra. Most of the rain away from the prominence can be considered to be optically thin in Mg II k (see Supplementary information). To retrieve the multiple components a multiple Gaussian fitting was performed automatically at every pixel and for every time step. The fitting routine acts based on various thresholds such as large enough signal-to-noise (SNR) ratio, sufficient data points to fit individual profiles and discards pixels hit by cosmic rays. First, a single Gaussian is fitted and the result is used as an initial guess for additional single and double Gaussian fits. The best fit is selected based on the lowest sigma errors and is then subtracted to the original spectral profile. Additional single Gaussian fits are performed on the residuals on the blue and red parts of the spectra to detect higher Doppler shifted components with high enough SNR that are not captured by the first fits. In this way a maximum of 4 possible components are allowed for each spectral profile. A check on the fitting routine was done based on an extensive visual inspection on random locations in time and space. A total of roughly 120,000 fits were obtained for the $\mathrm{Mg}$ II line with $60 \%$ found to be best represented by a single Gaussian with no other component. The rest was best represented with either a double Gaussian and/or a single Gaussian with additional Doppler components on the blue or red side of the spectrum. The Si IV spectra present in general a much lower SNR, leading to far fewer detections $(11,000)$. An additional check on the consistency of the results was then preformed by spatially binning along the slit by 3 pixels and remaking the analysis. 
Statistical evolution of the nanojets' spectral properties. The Doppler and non-thermal velocities show a significant variation during the expansion ( $t=84 \mathrm{~min}$ to $97 \mathrm{~min})$ in both the $\mathrm{Mg}$ II $\mathrm{k}$ and Si IV 1402.77 lines (Supplementary Fig. 13), with an increase of the Doppler velocities mainly towards the blue reaching speeds of $150 \mathrm{~km} \mathrm{~s}^{-1}$, and a corresponding increase of the non-thermal velocities up to $80 \mathrm{~km} \mathrm{~s}^{-1}$. The peak and integrated intensities increase by more than 2 orders of magnitude above the background noise in $\mathrm{Si} \mathrm{IV}$, while in $\mathrm{Mg}$ II the profiles have increased integrated intensities and reduced peaks, indicating heating of the initially cool material.

The observed properties of the jets indicate that we can define them based on their dynamics and Si IV intensities. We define a jet as an event satisfying both a large enough integrated intensity in the Si IV line (set to $200 D N \approx 1.4$ times the average rain emission during the period prior to the loop expansion) and a large enough summed Doppler velocity and non-thermal velocity (where the sum occurs also over all spectral components, and we set this velocity threshold to $100 \mathrm{~km} \mathrm{~s}^{-1}$ ). This choice is based on the distribution in total intensity and velocity of the IRIS spectra, shown in Extended Data Fig. 7. This nanojet definition is also supported by our numerical simulation results. We find 445 spectra (slit pixels) satisfying these conditions, all happening during the last 4 min of observation (Extended Data Fig. 7 and Table 1). The nanojets appear as highly localised features along the slit (marked with arrows in the Figure), first towards the apex and expand rapidly along and down the loop leg. Despite the countable nature observed in Figs. 2, 3 and 4 (and in Extended Data Figs. 1, 2 and Supplementary Figs. 2-9, 16 and 17), determining the precise number of detected nanojets in this dataset is not straightforward, due to their tight clustering and very fast nature. Taking an average width of a nanojet of $\approx 500 \mathrm{~km}$ and an average lifetime of $15 \mathrm{~s}$ (about 
10 measurements per nanojet with the IRIS slit, see Extended Data Fig. 7, panels c), we estimate a nanojet number of 44 captured by the IRIS slit. Assuming a constant occurrence rate over the time interval where they manifest we estimate the total number of nanojets above 150 . However, this is very likely a lower threshold given their clustering, avalanche-like occurrence and the limits of current detectability.

DEM analysis. The thermal evolution of the coronal plasma can be constrained by the AIA observations. Here we derive the differential emission measure (DEM) by applying the inversion method of [36], to the timeseries of the coronal AIA passbands (see Supplementary information). The loop can be distinguished in the emission measure $(E M)$ plots of Extended Data Fig. 9 (panel a) in a wide range of temperatures, from $\log T=5.5$ (corresponding to the cool EUV material surrounding the condensations), to $\log T=6.7$ and possibly higher. The fact that the pixels along the loop are consistently showing the same temperature range provides support for the presence of these temperatures. The appearance of hot plasma emission in the $\operatorname{loop}(\log T=6.3-6.5$ and possibly higher) is evidenced in the EM difference image (Extended Data Fig. 9, panel b).

The observed loop strand in the EUV channel has an average thickness of $1400 \mathrm{~km}$. Taking a depth for the structure similar to that in the POS and average $E M$ values of $10^{28}-10^{28.7} \mathrm{~cm}^{-5}$ we obtain electron number densities for the EUV emitting material in the range $[8.5-19] \times 10^{9} \mathrm{~cm}^{-3}$. The jets, having an average width of $500 \mathrm{~km}$, have number densities in EUV in the range [1.4 3.1] $\times 10^{10} \mathrm{~cm}^{-3}$. Note that these densities are close but smaller than the densities of the rain strands (Supplementary information). It is likely that due to their very small size the jets fail to make a significant deviation to higher $E M$ values. This small difference can also be attributed to 
the compressibility of the plasma in the reconnection region. The fact that the nanojets are mostly absent in the IRIS $2796 \AA$ A filter also supports a difference in density.

Evolution of braiding and twist of the loop structure. The IRIS and Hinode observations show several coronal rain strands at high resolution crossing each other near the apex of the loop. The misalignment between the strands can be seen in Extended Data Fig. 10 and amounts to an apparent maximum in the POS of $25^{\circ} \pm 5^{\circ}$. The location of the crossing in the POS of strands near the apex seems to coincide with the first location of the nanojet occurrence, intensity brightening and plasmoid ejection. Previously red-shifted downflowing plasma is replaced by rapidly evolving blue-shifted strands moving at total speeds of $\approx 60 \mathrm{~km} \mathrm{~s}^{-1}$ towards the loop apex. This motion is then replaced by consistently redshifted and blue-shifted strands towards the end of the observation (see $\mathbf{g}, \mathbf{h}$ panels in Extended Data Fig. 10 and the blue/red arrows in Supplementary Fig. 14), respectively at the lowest and highest portions of the loop, suggesting an azimuthal motion (Supplementary Fig. 14) and a complex untwisting motion of the loop. At the same time the loop structure expands, becomes thinner in all spectral channels and the angle between the helical shape of strands and the loop axis is reduced to $10^{\circ} \pm 3^{\circ}$. These features suggest an overall reduction in the braiding of the loop (see animation of Extended Data Fig. 10).

The nature of the nanojets. The dynamics of the nanojets can be understood from component magnetic reconnection between magnetic field lines from below the loop with the field lines connected to the prominence above (Supplementary information, Supplementary Fig. 15). In this scenario the expected reconnection outflow is partly in the POS, being perpendicular to the rain strands, with a component along the LOS that is determined by the presence of shear and twist. 
Since the observed LOS velocity component of the jets is on the same order as the POS component we expect a sheared component of the field of the same order as the misalignment in the POS (in the range $10^{\circ}-30^{\circ}$ ). We therefore interpret the nanojet as a part of the reconnection outflow, with magnetic tension being the driver of the observed dynamics. This kind of nanojet is different from the usual reconnection-driven jet concept in the sense that the observed outflow is not directed along the guide field. This is supported by the fact that both, the nanojet axis and the ejected plasmoids are perpendicular to the loop (Fig. 2), and trace new, rapidly separating strands. Numerical work has shown that plasmoids produced by the tearing mode merge in the reconnection exhaust to form larger and slower plasmoids [37]. It is therefore likely that the observed plasmoids are part of the upper tail of the size and energy distribution for such reconnection events.

A peculiar aspect of the nanojets is that almost all point radially inward with respect to the curvature of the loop (for an exception see nanojet N12, occuring further down the leg, Supplementary Fig. 8). This can be explained by the loop curvature, which implies that magnetic tension is expected to point mostly inward. Indeed, taking a circular geometry, the ratio of inward to outward magnetic tension between 2 slightly misaligned strands can be as high as 100 with the significant possibility of no outward component (thus no outward jet). On average we therefore expect either only inward jets or faster and longer inward jets (and therefore more visible). Also, contrary to the inward jets, the outward jets, if any, have the loop as background emission and are therefore much harder to detect. In this case, since all inward jets are mostly blue-shifted we expect the respective outward components to be redshifted. This is supported by the presence of redshifted secondary components for some nanojets (as seen in Extended Data Fig. 2 and Supplementary Fig. 5) and the 
fact that the non-thermal broadening of the jet spectra is of the same order of magnitude as their Doppler shifts.

Reports of other jets in the solar corona exist $[18,38]$, with a transverse morphology to the coronal structures, episodic and of singular nature (i.e. non bi-directional). Their generation mechanism could have common features with the nanojet's, although they involve different topologies and an energy range significantly higher than the nanojet and nanoflare's scenario.

Energetics. Based on the observed characteristics we can estimate the rate of kinetic and thermal energy outflow in the nanojet. We can further estimate the total energy liberated during a single event. Taking a representative total outflow speed of $v_{o}=100 \mathrm{~km} \mathrm{~s}^{-1}$, inflow densities determined by EUV absorption of $\rho_{i}=\left[1.6 \times 10^{10}-1.6 \times 10^{11}\right] \mathrm{cm}^{-3}$, outflow densities determined by DEM analysis of $\rho_{o}=[1.4-3.1] \times 10^{10} \mathrm{~cm}^{-3}$, an outflow width $\ell$ on the same order as the width of the nanojet (with a representative value of $\ell=500 \mathrm{~km}$ ) and an average temperature of $2 \mathrm{MK}$, we obtain an outflow kinetic energy rate of $\left[5.8 \times 10^{14}-1.29 \times 10^{15}\right] \mathrm{erg} \mathrm{cm}^{-1} \mathrm{~s}^{-1}$ and an outflow thermal energy rate of $\left[2.9 \times 10^{15}-6.4 \times 10^{15}\right] \mathrm{erg} \mathrm{cm}^{-1} \mathrm{~s}^{-1}$. Taking a representative time span of $15 \mathrm{~s}$ and a representative length of $1,500 \mathrm{~km}$ we estimate the total energy released by a nanojet to be $[7.8-17.3] \times 10^{24} \mathrm{erg}$. We expect that the observed range of energies corresponds to the high end of the nanojet distribution, most of which should be unresolved by the present observations.

Numerical modelling of nanojets. To better understand the dynamics of the nanojet generation in the solar corona, we devise non-ideal MHD simulations where two adjacent and parallel, gravitationally stratified coronal loops are slightly tilted, in accordance with the observed small POS 
crossings between rain strands.

The setup we use here is inspired from $[39,40]$. The initially magnetostatic loops include a chromosphere-like layer, a transition region and a million degree corona, relaxed to a steady state by solving the 3D resistive MHD equations with the PLUTO code [41]. The MHD equations include thermal conduction and radiative losses, and incorporate an anomalous magnetic resistivity term that switches on to (otherwise) non-zero values whenever the current density exceeds a specific threshold (see Supplementary Information).

We impose a transient driver at the chromospheric footpoints of both loops that slowly drifts them in opposite directions, such as to generate a slight $\mathrm{x}$-type misalignment in the corona. The rearrangement of the magnetic field in the corona leads to a steady increase of the misalignment angle $\theta_{t}$ between both loops, from $3^{\circ}$ at $t \approx 180$ s to almost $8^{\circ}$ at $t \approx 360$ s (Supplementary Fig. 19, panel b), before slightly decreasing.

Near $t=380 \mathrm{~s}$, the increase of the tilt angle produces an increase of the electric current between the loops that overcomes the threshold for the anomalous resistivity and the magnetic field diffusion sets in (Supplementary Fig. 19, panel a). At this time, the characteristic length $L$ of the region in which the electric currents are larger than the threshold is about $0.3 \mathrm{Mm}$, the plasma velocity $V$ is of the order of $50 \mathrm{~km} \mathrm{~s}^{-1}$, and the resistivity coefficient is $\eta_{0}=10^{14} \mathrm{~cm}^{-2} \mathrm{~s}^{-1}$. This leads to a magnetic Reynolds number of

$$
R_{M}=\frac{V L}{\eta_{0}} \sim 1
$$


In this regime the time scales for the diffusion and advection of the magnetic field are similar and magnetic reconnection sets in. Magnetic field lines change connectivity from one loop to the other, with a change of direction at the centre of the domain (the $B_{y}$ component of the magnetic field grows up to $0.07 \mathrm{G}$ before flipping sign after the reconnection). This changed topology leads to an enhanced magnetic tension in that region (Supplementary Fig. 20, panel b), which starts displacing the plasma transversely. Accordingly, the velocity $V_{y}$ of the plasma rapidly increases well above $200 \mathrm{~km} \mathrm{~s}^{-1}$ (Supplementary Fig. 20, panel c), which is much higher than any value previously found in the simulation.

Near the centre of the domain the magnetic energy initially increases with the $y$-component of the local magnetic field (Supplementary Fig. 19, panel b). When the anomalous resistivity is triggered, the magnetic energy drops to a value lower than the initial one, and the thermal and kinetic energies increase on a very similar timescale and magnitude (Supplementary Fig. 21, panel a). The large variation of magnetic and thermal energies is mainly due to driving 2 large flux tubes (leading to a large Poynting flux from the lower boundaries). On the other hand, the kinetic energy increase appears smaller because is more localised and is partly converted into thermal energy during the plasma compression. This is associated with an average speed of $\sim 140 \mathrm{~km} \mathrm{~s}^{-1}$, whereas the plasma is accelerated locally up to $\sim 300 \mathrm{~km} \mathrm{~s}^{-1}$. We identify as jets the localised regions near the centre of the domain where the plasma is accelerated to $200 \mathrm{~km} \mathrm{~s}^{-1}$ or more (Fig. 5). The temperature experiences a jump of $3 \times 10^{6} \mathrm{~K}$ when the reconnection is triggered (Supplementary Fig. 21, panel b). The outflow thermal energy rate is comparable to the inflow electromagnetic energy rate (Supplementary Fig. 21, panel c). Importantly, the value of the critical 
current above which the anomalous resistivity sets in does not influence the dynamics of the nanojet and the attained temperature (see Supplementary Information).

The region where the electric current is equal to the threshold to trigger the anomalous resistivity has an X-shape located between the two loops (Fig. 5b). The highest velocities are highly localised, where the region with $\left|V_{y}\right| \geq 190 \mathrm{~km} \mathrm{~s}^{-1}$ is a few Mm long (Fig. 5c). However, $V_{y}$ velocities around $100 \mathrm{~km} \mathrm{~s}^{-1}$ are also found along the reconnected field lines to a distance half-way down the loop, indicating a strong transverse motion of the entire reconnecting strand. Importantly, the high velocity region is adjacent to the reconnected magnetic field lines that show near $z=0$ a curvature generating an outward magnetic tension (Supplementary Fig. 20). Such configuration is not found before reconnection. The longitudinal velocities $\left(V_{z}\right)$ are always below a few tens of $\mathrm{km} \mathrm{s}^{-1}$. Hence, the reconnection triggers the highest velocities mostly along the $y$-direction, i.e. perpendicular to the guide magnetic field and coronal loop axes, and only minor motions along the loops.

Therefore, the numerical model leads to results with the same telltale signatures as those of the observed nanojets. On the other hand, the timescale of the changes and corresponding energy release is large due to the size of the flux tubes being driven. The changes occur in a timescale of $100 \mathrm{~s}$, longer than the timescale of a single nanojet (15 s or less) but similar to that of the nanojet clusters. Also, the localisation of the jets in the numerical model is roughly 5 times larger than that of a single nanojet, but similar to the width of the nanojet cluster. Although our model does not capture the exact conditions of the observed plasma (partially ionised, cool and dense), we consider that the same physics would occur in more detailed experiments. The differences with the 
numerical experiment, in particular, the single and clustered manifestation of the nanojets at faster timescales and smaller length scales, accompanied by plasmoids, constitute a challenge for future numerical models of magnetic reconnection that can clarify the detailed physics of this process in the solar corona.

\section{Methods References}

36. Cheung, M. C. M. et al. Thermal Diagnostics with the Atmospheric Imaging Assembly on board the Solar Dynamics Observatory: A Validated Method for Differential Emission Measure Inversions. Astrophys. J. 807, 143 (2015).

37. Huang, Y.-M. \& Bhattacharjee, A. Distribution of plasmoids in high-lundquist-number magnetic reconnection. Phys. Rev. Lett. 109, 265002 (2012).

38. Chen, H., Zhang, J., Ma, S., Yan, X. \& Xue, J. Solar Tornadoes Triggered by Interaction between Filaments and EUV Jets. Astrophys. J. Lett. 841, L13 (2017).

39. Petralia, A., Reale, F., Orlando, S. \& Testa, P. Bright Hot Impacts by Erupted Fragments Falling Back on the Sun: Magnetic Channelling. Astrophys. J. 832, 2 (2016).

40. Petralia, A., Reale, F. \& Testa, P. Guided flows in coronal magnetic flux tubes. Astron. Astrophys. 609, A18 (2018).

41. Mignone, A. et al. The PLUTO Code for Adaptive Mesh Computations in Astrophysical Fluid Dynamics. Astrophys. J. Suppl. 198, 7 (2012). 
Data availability statement The IRIS and Hinode observations used in the instrument data figures are available at https://bit.ly/3gcI2Wt. Other data used in this article are available upon reasonable request.

Code availability statement PLUTO is a modular Godunov-type code to solve mixed hyperbolic/parabolic systems of partial differential equations (conservation laws) targeting high Mach number flows in astrophysical fluid dynamics. Equations are discretized and solved on a structured mesh that can be either static or adaptive through the Adaptice Mesh Refinement (AMR) interface. PLUTO is distributed freely under the GNU general public license and can be downloaded at http://plutocode.ph.unito.it/. 